Chinese Journal of Organic Chemistry

\title{
无机盐碘化钠光促炔烃双碘化
}

\author{
鲁玲玲 ${ }^{a}$ 李一鸣 ${ }^{a}$ 姜雪峰*,a,b \\ ( ${ }^{a}$ 华东师范大学化学与分子工程学院 上海市绿色化学与化工过程绿色化重点实验室 上海 200062) \\ ( ${ }^{b}$ 南开大学化学学院 元素有机化学国家重点实验室 天津 300071)
}

\begin{abstract}
摘要 1,2 -双碘取代烯烃，因具有高度官能团衍生性，可作为多种杂环药物和有机共轭材料功能分子的合成前体. 本 工作以稳定易得的无机盐碘化钠作为碘源, 空气作为氧化剂, 仅仅在常温常压可见光(蓝光)照射下, 即可将炔烃高选择 性且方便地转化为反式 1,2-双碘烯烃化合物. 反应条件温和, 试剂廉价易得, 操作简便稳定, 无需额外添加其他过渡金 属催化剂或氧化试剂. 同时, 该法底物普适性优秀, 端炔、内炔均适用, 并且对于多种含杂原子与活性氢的肽或糖类结 构也能优秀地兼容
\end{abstract}

关键词 1,2-双碘烯烃; 炔烃; 双碘化; 可见光

\section{Visible-Light-Promoted Diiodination of Alkynes Using Sodium lodide}

\author{
$\mathrm{Lu}_{\text {, Lingling }}{ }^{a} \quad \mathrm{Li}_{\text {, Yiming }}{ }^{a} \quad$ Jiang, Xuefeng ${ }^{*, a, b}$ \\ $\left({ }^{a}\right.$ Shanghai Key Laboratory of Green Chemistry and Chemical Process, School of Chemistry and Molecular Engineering, \\ East China Normal University, Shanghai 200062) \\ $\left({ }^{b}\right.$ State Key Laboratory of Elemento-organic Chemistry, Nankai University, Tianjin 300071)
}

\begin{abstract}
Diiodoalkenes can be used as precursors for synthesis of functional molecules such as heterocyclic drugs and organic conjugated materials due to their derivability of functional groups. Herein, alkynes can be converted into 1,2-transdiiodioalkenes efficiently and conveniently by using inexpensive and stable sodium iodide as iodine source and air as oxidant under the visible-light (blue light) with normal temperature and atmospheric pressure. The corresponding reactions were operated under mild conditions with inexpensive and easily accessible reagents, which obviate the need of transition-metal-catalysts or oxidizing reagents. Meanwhile, this method is compatible with a wide range of substrates, including terminal and internal alkynes even the peptide and carbohydrates containing a variety of heteroatoms and active hydrogen.

Keywords 1,2-diiodoalkenes; alkynes; diiodination; visible-light
\end{abstract}

有机卤化物是合成化学应用最广的合成子之一, 其 中尤属碳碘键反应活性最好, 广泛应用于医药和材料的 合成中，例如交叉偶联建立药物结构的链接与安装 ${ }^{[1]}$ 、 构建光电材料共轭电子传输体系 ${ }^{[2]}$ 、碘锂交换后気化形 成代谢性大幅改善的氛代药物 ${ }^{[3]}$, 以及 $I^{127}$ 同位素在医 学诊断中放射性同位素标记的造影剂 ${ }^{[4]}$ 等. 2019 年, 受 卤化酶 ${ }^{[5]}$ 工作机制启发, 我们小组 ${ }^{[6]}$ 报道了一种新型炔 烃氧化偕二卤化反应: 以简单易得的无机卤盐 $(\mathrm{LiCl}$ 和 $\mathrm{NaBr}$ ) 为卤源, 通过可见光照射, 于常温常压下便实现 了炔烃向 $\alpha, \alpha$-偕二卤苯乙酮类化合物的高效转化. 在此 反应过程中, 底物自催化实现光激发, 实现卤离子与氧
气的双重活化, 生成的卤自由基进一步被氧化生成卤正 离子, 进而与炔烃形成卤鎓阳离子中间体, 经历水的亲 核进攻生成烯醇中间体，再次卤化、去质子化得到 $\alpha, \alpha$ 偕二卤苯乙酮类化合物. 我们猜想, 假若体系中存在另 一种亲核性更强的化合物, 就会阻断水的进攻而经历其 他转化路径. 基于我们小组 “从无机盐向有机物转化” 的理念，采用廉价易得的无机盐碘化钠作为碘源，在类 似的条件下却生成了反式 1,2-双碘烯烃类化合物, 而未 发现与氯和溴相似的结果 (Scheme 1A). 其原因可能是: (1)从原子半径来看, 碘原子具有相较氯、澳 $(0.097 \mathrm{~nm}$, $0.112 \mathrm{~nm}$ )更大的半径 $(0.132 \mathrm{~nm})$, 可极化度大, 所形成

* Corresponding author. E-mail: xfjiang@chem.ecnu.edu.cn

Received May 23, 2020; revised June 26, 2020; published online July 15, 2020.

Dedicated to Professor Henry N. C. Wong on the occasion of his 70th birthday.

Project supported by the National Key Research and Development Program of China (No. 2017YFD0200500), and the National Natural Science Foundation of China (Nos. 21971065, 21722202, 21672069).

国家重点研发计划(No. 2017YFD0200500)、国家自然科学基金(Nos. 21971065, 21722202, 21672069)资助项目. 
(A) Oxidative halogenation

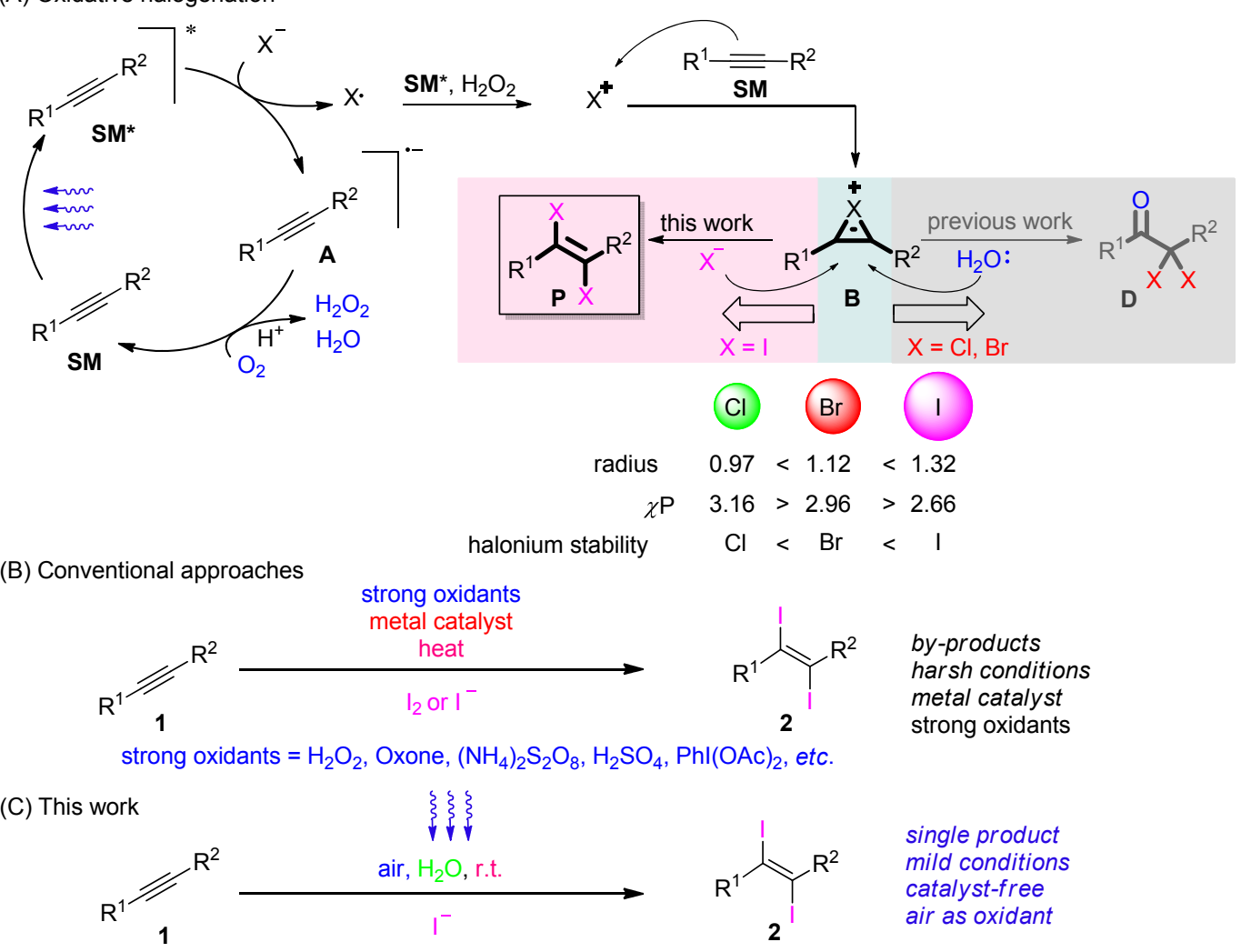

图式 1 炔烃的双碘化

Scheme 1 Diiodination of alkynes

鎓离子中间体更稳定; (2)从电负性来看, 碘原子具有相 较氯、溴 $(3.16,2.96)$ 较小的电负性 $(2.66)$, 且亲核性强于 水.

反式 1,2-双碘烯烃类化合物因其高度的官能团衍生 性，广泛地用于取代炔烃 ${ }^{[7]}$ 、取代烯烃 ${ }^{[8]}$ 、羧酸酯 ${ }^{[9]}$ 、呋

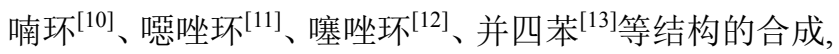
亦可作为共轭有机光电材料核心骨架 ${ }^{[14]}$. 基于其广泛 用途, 化学家们开发出了多种制备方法(Scheme 1B): 最 直接的制备方式是以碘单质 $\left(\mathrm{I}_{2}\right)$ 作为碘源与炔烃反应, 但由于反应速率慢、效率低, 通常需要额外使用较多的 氧化试剂 ${ }^{[15]}$ 、金属试剂辅助催化氧化 ${ }^{[16]}$ 或加热等 ${ }^{[16 a, 17]}$ 方式促进反应进行; 另一种是以 $\mathrm{I}^{-}$为碘源的氧化碘化 法, 通过添加适当的氧化剂及催化剂, 原位氧化 $\mathrm{I}^{-}$生成 亲电碘对炔烃进行碘化. 常用氧化剂有 $O x o n e^{[18]}$ 、 $\mathrm{H}_{2} \mathrm{SO}_{4}{ }^{[19]} 、\left(\mathrm{NH}_{4}\right)_{2} \mathrm{~S}_{2} \mathrm{O}_{8}{ }^{[20]} 、 \mathrm{PhI}(\mathrm{OAc})_{2}{ }^{[21]}$ 等, 然而, 过量 氧化剂的使用往往伴随着其它副产物的生成而导致产 率低, 以及底物官能团兼容性差等问题. 因此, 发展一 种温和条件下实现单一选择性 1,2-双碘烯烃类化合物的 合成显得尤为重要. 在此, 我们报道以炔烃为原料及自 催化剂, 无机盐碘化钠为碘源, 自然条件(可见光、空 气、水、常温、常压)下实现反式 1,2-双碘烯烃类化合物 的合成(Scheme 1C), 为高效、绿色、可持续发展的化学
提供一种新的选择.

\section{1 结果与讨论}

\section{1 反应条件的篮选}

我们以苯乙炔 $1 \mathrm{a}$ 为模板底物, 碘化钠作为碘源, 乙 腈作为溶剂进行条件篮选(表 1). 考虑到碘化钠作为无 机盐的溶解性问题, 尝试向反应体系中添加水来改善其 在体系中的溶解情况, 其中以 20 equiv.为最优. 在蓝光 照射及空气氛围中, 添加 Brönsted 酸 $\mathrm{NaHSO}_{4} \cdot \mathrm{H}_{2} \mathrm{O}$ 时能 以 $90 \%$ 的分离收率得到目标产物 1,2-双碘乙烯基苯 2a(表 1, Entry 1). 但将 Schlenk 反应管关闭隔绝空气后, 反应产率大幅下降至 56\%(表 1, Entry 2), 若在氮气氛围 下，反应则不能发生(表 1, Entry 3). 这些说明: 该反应 需要氧气, 并且 $25 \mathrm{~mL}$ 的 Schlenk 管中原有空气所含氧 气量不足以使反应完全转化. 当使用碘化钾或碘化铵代 替碘化钠作为碘源时, 产率有所降低(表 1, Entries 5 和 6). 此外，当反应在室温下避光搅拌 $10 \mathrm{~h}$ 反应不能发生， 即使在 $70{ }^{\circ} \mathrm{C}$ 的温度下进行，也仅仅能以 $28 \%$ 产率得到 目标化合物(表 1, Entries 7,8). 验证实验表明质子酸是 必要的, 可能用于帮助产生 $\mathrm{H}_{2} \mathrm{O}_{2}$ 促进反应体系循环(表 1, Entries 9，11), 如果反应体系没有水(表 1 , Entry 10), 产率则下降到 $40 \%$. 因此最终确定反应最佳条件为: 3.0 
equiv. 碘化钠为碘源, 4.0 equiv. $\mathrm{NaHSO}_{4} \cdot \mathrm{H}_{2} \mathrm{O}$ 为添加剂, 20.0 equiv.水为共溶剂, 乙腈为溶剂, 蓝光照射, 空气氛 围, 室温搅拌.

表 1 双碘化反应条件优化 ${ }^{a}$

Table 1 Optimization of diiodization reaction conditions

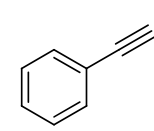

1a

Entry Variation from standard conditions

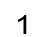

2

3

4

5

\section{6}

7

\section{8}

9

10

11

1

None

None $^{d}$

$\mathrm{N}_{2}$ instead of air

$\mathrm{O}_{2}$ instead of air

$\mathrm{KI}$ instead of $\mathrm{Nal}$

$\mathrm{NH}_{4} \mathrm{l}$ instead of Nal

No light

No light, $70^{\circ} \mathrm{C}$

No $\mathrm{NaHSO}_{4} \cdot \mathrm{H}_{2} \mathrm{O}$

$\mathrm{No} \mathrm{H}_{2} \mathrm{O}$

Only Nal

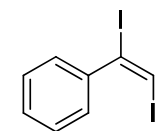

$2 a$

${ }^{a}$ The reaction conditions: 1a $(0.2 \mathrm{mmol}), \mathrm{NaI}(0.6 \mathrm{mmol}), \mathrm{NaHSO}_{4} \cdot \mathrm{H}_{2} \mathrm{O}(0.8$ $\mathrm{mmol}), \mathrm{H}_{2} \mathrm{O}(4.0 \mathrm{mmol}), \mathrm{MeCN}(2.0 \mathrm{~mL})$, room temperature, air, $2 \mathrm{~W} \times 3$ blue LEDs, 10 h. ${ }^{b} \mathrm{NMR}$ yield with $\mathrm{CH}_{2} \mathrm{Br}_{2}$ as internal standard. ${ }^{c}$ Isolated yields. ${ }^{d}$ Schlenk tubes $(25 \mathrm{~mL})$ were sealed.

\section{2 底物普适性考察}

在最优条件下, 对炔烃的双碘化反应进行了底物普 适性考察(表 2). 当底物苯环上有甲基、乙基、叔丁基、 正戊基供电性烷基取代时, 均能以优秀的产率得到目标 化合物 ( $2 \mathrm{~b} \sim 2 \mathrm{e})$. 即使是强供电性基团如甲氧基时, 反 应仍能以良好的产率生成 1,2-双碘化产物(2f, 2g). 当苯 环上不同位点被卤原子取代( $\mathbf{2} \mathbf{h} \sim \mathbf{2 k})$ 时，反应也能以良 好的产率顺利进行. 对位苯基取代的炔烃在该条件下也 能以 70\%的产率得到目标产物(21). 此外, 缺电性的吡 啶环也能在该反应体系下兼容, 尽管产率不高 $(\mathbf{2 m})$. 除 端炔外, 内炔底物也能以中等到良好的产率得到相应的 目标产物( $20 \sim 2 q)$. 对芳基炔烃底物进行尝试之后, 我 们对烷基炔烃底物也进行了考察, 发现无论是链状的炔 烃, 或是含有敏感基团(酯基、羟基)的底物, 反应均能以 中等到良好的产率得到 1,2-双碘化产物 $(2 \mathbf{r} \sim 2 \mathbf{w})$. 含有 酰胺 $(2 \mathrm{n}, 2 \mathrm{x})$ 或磺酰胺 $(2 \mathrm{y})$ 基团的底物在该反应体系下 也能正常反应, 不受影响. 肽类、糖类和萜类结构单元 不仅广泛存在于天然产物和各类生命分子中, 也常常被 引入到药物结构中. 我们考察了这类结构单元在该反应
体系中的兼容性情况. 首先对含有酰胺键(肽键)的二肽 类底物进行尝试, 发现能以较低产率得到双碘化产物 (2z). 值得一提的是, 葡萄糖衍生的底物结构在体系中 也能兼容(2aa). 此外, 对于萜类化合物如合成右旋龙脑 衍生的底物能以 $89 \%$ 的产率得到双碘化产物(2ab). 1,4二苯基-1,3-丁二炔在该体系中并不能被有效转化, 原 料几乎全部剩余.

\section{3 可能的反应机理}

当使用邻位苯甲酸酯基取代的底物 $\mathbf{S}$ 在标准条件下 反应时得到环化产物 $\mathbf{S}^{\prime}$, 我们推测其是炔烃与体系中 $\mathrm{I}^{-}$ 氧化得到的 $\mathrm{I}^{+}$形成碘鎓离子后再被邻位的酯基亲核进 攻所致(Scheme 2A). 因此, 我们认为反应过程中的碘以 $\mathrm{I}^{+}$的形式与炔烃反应. 该反应体系在室温无光条件下是 不反应的, 加热到回流条件下也仅有 $28 \%$ 的转化(表 1 , Entry 8), 所以在标准条件下, 光是起了关键作用的; 结 合我们之前的研究, 苯乙炔的氧化电势是高于碘负离子 的, 因此受光激发的苯乙炔可能帮助更加有效地完成电 子传递，提升整个体系的反应效率. 基于上述的实验结 果以及氧卤化 ${ }^{[6]}$ 的工作基础, 我们提出了如下可能的反 应机理(Scheme 2B): 首先，具有光敏特性的苯乙炔在可 见光作用下被激发, 并从 $\mathrm{I}^{-}$得到一个电子, 生成苯乙炔 阴离子自由基 $\mathbf{A}$ 和碘自由基 $\mathrm{I}^{-}$, 而这种自由基缓释的方 式使该碘化反应具有了十分优异的底物兼容性. 在质子 的辅助下, 空气氧化 $\mathbf{A}$ 生成过氧化氢或水, 并再生基态 苯乙炔. 然后, 碘自由基 I 被激发态底物(SM*)或过氧 化氢氧化成碘正离子 $\mathrm{I}^{+}$. 随后 $\mathrm{I}^{+}$与底物 $\mathbf{S M}$ 加成生成碘 鎓离子中间体 B. 与氧澳、氯化反应不同的是，碘鎓离 子中间体 $\mathbf{B}$ 并没有被水进攻，而是被 $\mathrm{I}^{-}$进攻生成最终产 物 $\mathbf{P}$, 这可能是由于 $\mathrm{I}^{-}$的亲核性远强于 $\mathrm{H}_{2} \mathrm{O}$ 导致的. 尽 管如此, 我们发现碘负离子溶液或固体在自然条件下接 触酸后颜色会变深, 相关颜色可能来自于碘单质, 因此 我们并不能排除自然条件下碘负离子被氧化而发生后 续转化的可能性.

\section{2 结论}

秉承 “无机卤向有机卤转化” 的理念, 我们报道了 在自然条件 (常温、常压、可见光、空气)下，以无机盐 碘化钠为碘源, 空气作为氧化剂, 运用苯乙炔类化合物 自身的光敏特性，实现了将炔烃高选择性、方便地转化 为反式 1,2 -双碘烯烃化合物. 该方法反应条件温和，试 剂廉价易得、操作简便, 无需额外添加其他过渡金属催 化剂或氧化试剂, 并且具有较好的底物普适性, 端炔内 炔均可适用, 对于多种含杂原子与活性氢的肽或糖类结 构均能优秀地兼容, 实现了许多传统碘化无法兼容的 体系，为未来的复杂体系应用奠定了基础. 
表 2 底物拓展 ${ }^{a}$

Table 2 Scope of substrates

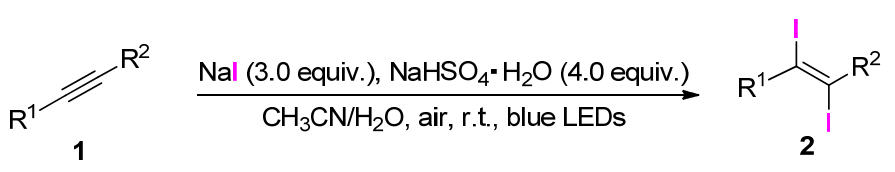<smiles>I/C=C(/I)c1ccccc1</smiles>

2a, $90 \%$

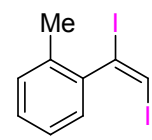

2b, $78 \%$<smiles>CCc1ccc(/C(I)=C\I)cc1</smiles>

2c, $92 \%$<smiles>CCCCCc1ccc(/C(I)=C\I)cc1</smiles>

2d, $90 \%$<smiles>CC(C)(C)c1ccc(/C(I)=C\I)cc1</smiles>

2e, $78 \%$<smiles>COc1cccc(/C(I)=C\I)c1</smiles>

2f, $75 \%$<smiles>COc1ccc(/C(I)=C\I)cc1</smiles>

2g, $70 \%$<smiles>Fc1cccc(/C(I)=C/I)c1</smiles>

2h, $59 \%$<smiles>Fc1ccc(/C(I)=C/I)cc1</smiles>

2j, $81 \%$<smiles>Brc1ccc(/C(I)=C\I)cc1</smiles>

$2 k, 82 \%$<smiles>I/C=C(/I)c1ccc(-c2ccccc2)cc1</smiles>

2I, $70 \%$<smiles>C=C(I)c1cccnc1</smiles>

2m, $40 \%$<smiles>CC1(C)C(C(=O)Nc2cccc(/C(I)=C\I)c2)C1(C)C</smiles>
2n, $61 \%$<smiles>CC(I)=C(I)c1ccccc1</smiles>

2o, $61 \%$<smiles>IC(CCc1ccccc1)=C(I)c1ccccc1</smiles>

2p, $85 \%$<smiles>Cc1ccc(/C(I)=C(\I)CCc2ccccc2)cc1</smiles>

2q, $64 \%$<smiles>I/C=C(/I)CCc1ccccc1</smiles>

2 r, $74 \%$<smiles>CC(Cc1ccc(C(C)(C)C)cc1)C(C)/C(I)=C\I</smiles>

2s, $40 \%$<smiles>COc1ccc(C(=O)OCC/C(I)=C\I)cc1OC</smiles>

2t, $77 \%$<smiles>CCCCCCCC/C(I)=C\I</smiles>

2u, $73 \%$<smiles>CC(I)=C(I)CO</smiles>

2v, $65 \%$<smiles>O=C(O)CCC/C(I)=C\I</smiles>

2w, $60 \%$

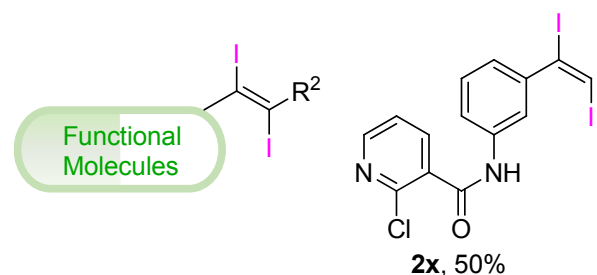

2x, $50 \%$<smiles>Cc1ccc(S(=O)(=O)Nc2cccc(/C(I)=C\I)c2)cc1</smiles>

2y, $72 \%$

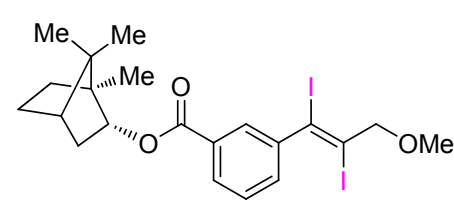

2ab, $89 \%$

${ }^{a}$ Reactions were performed with $1(0.2 \mathrm{mmol}), \mathrm{NaI}(0.6 \mathrm{mmol}), \mathrm{NaHSO}_{4} \mathrm{H}_{2} \mathrm{O}(0.8 \mathrm{mmol}), \mathrm{H}_{2} \mathrm{O}(4.0 \mathrm{mmol})$, and $\mathrm{MeCN}(2.0 \mathrm{~mL})$ at room temperature under air by $2 \mathrm{~W} \times 3$ blue LEDs irridation for $10 \mathrm{~h}$. Isolated yields.

(A) Byproduct analysis

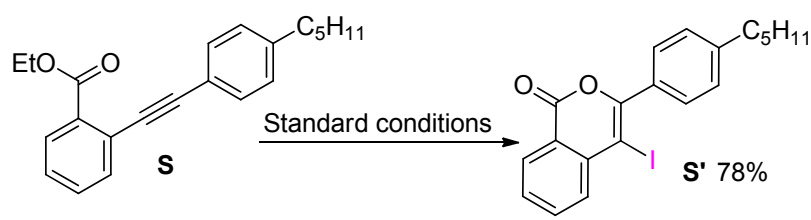

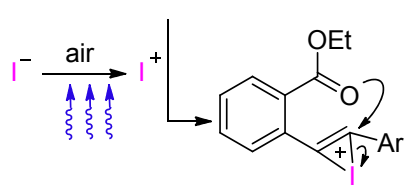<smiles>CCOc1ccc(C)cc1-c1ccc(OCC)c(Br)c1O</smiles>

(B) Proposed mechanism

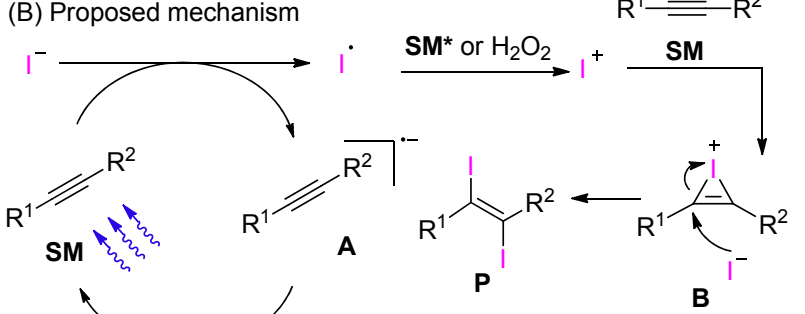

图式 2 可能的反应机理

Scheme 2 Proposed mechanism 


\section{3 实验部分}

\section{1 仪器与试剂}

核磁: Bruker AVANCE 400 型核磁共振波谱仪, 内 标为 TMS(四甲基硅烷), 氛代溶剂为 $\mathrm{CDCl}_{3}\left({ }^{1} \mathrm{H} \mathrm{NMR} \delta\right.$ : $7.26,{ }^{13} \mathrm{C}$ NMR $\left.\delta 77.0\right)$ 或 DMSO- $d_{6}\left({ }^{1} \mathrm{H}\right.$ NMR $\delta 2.50,{ }^{13} \mathrm{C}$ NMR $\delta 39.0$ ); 质谱: GCMS QP 2010 Ultra 和 HP 5989A 质谱仪; 红外: Bruker tensor (27)型红外光谱仪.

试剂: 反应所需的原料与溶剂均为商品化的化学纯 和分析纯试剂; 水为二次蒸馏水, 乙腈用 $\mathrm{CaH}_{2}$ 重蒸处 理. 柱层析使用的洗脱剂石油梄和乙酸乙酯均是经过重 蒸处理, 所用 $300 \sim 400$ 目柱层析硅胶为烟台江友硅胶 开发有限公司生产.

\section{2 实验方法}

将反应原料炔烃 $1(0.2 \mathrm{mmol})$ 、碘化钠 $(0.6 \mathrm{mmol})$ 、 一水合硫酸氢钠 $(0.8 \mathrm{mmol})$, 水 $(4.0 \mathrm{mmol})$ 加入到预先放 有磁力搅拌子的 Schlenk 反应管中, 加入 $2 \mathrm{~mL}$ 乙腈, 反 应管置于室温蓝光反应仪 $(2 \mathrm{~W} \times 3)$ 中, 开启光源、散热 器以及磁力搅拌, 保持反应管内部与外部大气相通, 反 应 $10 \mathrm{~h}$ 后, 用薄层色谱(TLC)板检测确认底物 1 完全转 化后, 用二氯甲烷稀释反应体系, 再加入无水硫酸镁干 燥, 过滤后将滤液用旋转蒸发仪除去有机溶剂后使用层 析柱分离纯化, 得到目标化合物 2 .

$(E)-\left(1,2\right.$-二碘乙烯基)苯 $(2 \mathrm{a})^{[21 b]}$ : 白色固体, $63 \mathrm{mg}$, 产率 90\%. m.p. $71.4 \sim 72.6{ }^{\circ} \mathrm{C}$ (lit. ${ }^{[21 b]} 71 \sim 73{ }^{\circ} \mathrm{C}$ ); ${ }^{1} \mathrm{H}$ NMR (400 MHz, Chloroform- $d$ ) $\delta: 7.38 \sim 7.33(\mathrm{~m}, 5 \mathrm{H})$, 7.27 (s, $1 \mathrm{H}) ;{ }^{13} \mathrm{C}$ NMR (101 MHz, Chloroform- $d$ ) $\delta: 143.1$, 128.9, 128.5, 128.4, 96.2, 80.8. MS (EI) $m / z: 356\left([\mathrm{M}]^{+}\right)$; IR (film) $v: 3061,2922,1481,1438,1234,1149,1072$, $837,781,759,694,665,597 \mathrm{~cm}^{-1}$.

(E)-1-(1,2-二碘乙烯基)-2-甲基苯(2b) ${ }^{[18]}$ : 黄色油状 液体, $58 \mathrm{mg}$, 产率 78\%. ${ }^{1} \mathrm{H}$ NMR $(400 \mathrm{MHz}$, Chloroform- $d$ ) $\delta: 7.25 \sim 7.16(\mathrm{~m}, 4 \mathrm{H}), 7.08(\mathrm{~d}, J=7.3 \mathrm{~Hz}, 1 \mathrm{H})$, 2.22 (s, $3 \mathrm{H}) ;{ }^{13} \mathrm{C}$ NMR (101 MHz, Chloroform- $d$ ) $\delta: 142.9$, 134.5, 130.6, 129.0, 127.6, 126.3, 96.2, 82.9, 19.5; MS (EI) $m / z: 370$ ([M] $]^{+}$); IR (film) $v: 3061,2916,1917,1477$, 1454, 1377, 1151, 1103, 1041, 846, 786, 748, 719, 657, $597 \mathrm{~cm}^{-1}$.

(E)-1-(1,2-二碘乙烯基)-4-乙基苯(2c $)^{[21 b]}$ : 黄色油状 液体, $71 \mathrm{mg}$, 产率 92\%. ${ }^{1} \mathrm{H}$ NMR $(400 \mathrm{MHz}$, Chloroform- $d$ ) $\delta: 7.33 \sim 7.29(\mathrm{~m}, 2 \mathrm{H}), 7.24(\mathrm{~s}, 1 \mathrm{H}), 7.20(\mathrm{~d}, J=$ $8.4 \mathrm{~Hz}, 2 \mathrm{H}), 2.67$ (q, $J=7.6 \mathrm{~Hz}, 2 \mathrm{H}), 1.27$ (t, $J=7.6 \mathrm{~Hz}$, $4 \mathrm{H}) ;{ }^{13} \mathrm{C}$ NMR (101 MHz, Chloroform- $d$ ) $\delta: 145.2,140.2$, 128.6, 127.8, 96.7, 80.1, 28.7, 15.1; MS (EI) $\mathrm{m} / \mathrm{z}: 384$ $\left([\mathrm{M}]^{+}\right)$; IR (film) $v: 3061,2962,1608,1498,1454,1408$,
1244, 1149, 1049, 850, 827, 775, $596 \mathrm{~cm}^{-1}$.

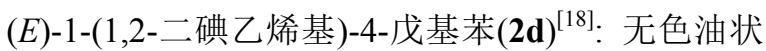
液体, $77 \mathrm{mg}$, 产率 90\%. ${ }^{1} \mathrm{H}$ NMR $(400 \mathrm{MHz}$, Chloroform- $d$ ) $\delta: 7.21(\mathrm{~d}, J=7.9 \mathrm{~Hz}, 2 \mathrm{H}), 7.15(\mathrm{~s}, 1 \mathrm{H}), 7.09(\mathrm{~d}$, $J=7.8 \mathrm{~Hz}, 2 \mathrm{H}), 2.56 \sim 2.50(\mathrm{~m}, 2 \mathrm{H}), 1.60 \sim 1.53(\mathrm{~m}, 2 \mathrm{H})$, $1.30 \sim 1.24(\mathrm{~m}, 4 \mathrm{H}), 0.83(\mathrm{t}, J=6.2 \mathrm{~Hz}, 3 \mathrm{H}) ;{ }^{13} \mathrm{C}$ NMR (101 MHz, Chloroform- $d$ ) $\delta: 144.1,140.2,128.5,128.3$, 96.7, 35.8, 31.5, 30.8, 22.5, 14.0; MS (EI) $m / z: 426\left([\mathrm{M}]^{+}\right)$; IR (film) v: 2953, 2924, 2854, 1487, 1442, 1116, 1028, $831,754,692,646,574 \mathrm{~cm}^{-1}$.

(E)-1-(叔丁基)-4-(1,2-二碘乙烯基)苯(2e $)^{[18]}$ : 淡黄 色油状液体, $64 \mathrm{mg}$, 产率 $78 \%$. ${ }^{1} \mathrm{H}$ NMR $(400 \mathrm{MHz}$, Chloroform- $d$ ) $\delta: 7.37$ (d, $J=8.4 \mathrm{~Hz}, 2 \mathrm{H}), 7.32$ (d, $J=8.2$ $\mathrm{Hz}, 2 \mathrm{H}), 7.23$ (s, 1H), 1.33 (s, 9H); ${ }^{13} \mathrm{C}$ NMR (101 MHz, Chloroform- $d$ ) $\delta$ : 152.1, 139.8, 128.3, 125.2, 96.7, 34.8, 31.2; MS (EI) $m / z: 412$ ([M] $]^{+}$); IR (film) $v: 2960,1606$, 1498, 1460, 1361, 1267, 1155, 1105, 1016, 856, 839, 819, $775,705,599 \mathrm{~cm}^{-1}$.

(E)-1-(1,2-二碘乙烯基)-3-甲氧基苯(2f) ${ }^{[21 b]}$ : 黄色油 状液体, $58 \mathrm{mg}$, 产率 $76 \%$. ${ }^{1} \mathrm{H}$ NMR $(400 \mathrm{MHz}$, Chloroform- $d$ ) $\delta: 7.34 \sim 7.22(\mathrm{~m}, 2 \mathrm{H}), 6.95(\mathrm{~d}, J=7.7 \mathrm{~Hz}, 1 \mathrm{H})$, $6.87(\mathrm{~d}, J=5.7 \mathrm{~Hz}, 2 \mathrm{H}), 3.83$ (s, 3H); ${ }^{13} \mathrm{C}$ NMR $(101 \mathrm{MHz}$, Chloroform- $d$ ) $\delta: 159.2,144.2,129.5,120.8,114.8,113.8$, 95.8, 55.3; MS (EI) $m / z: 386$ ([M] $]^{+}$); IR (film) $v: 3062$, 2920, 1589, 1577, 1477, 1427, 1286, 1263, 1220, 1047, $790,759,696 \mathrm{~cm}^{-1}$.

(E)-1-(1,2-二碘乙烯基)-4-甲氧基苯(2g $)^{[18]}$ : 无色油 状液体, $55 \mathrm{mg}$, 产率 70\%. ${ }^{1} \mathrm{H}$ NMR $(400 \mathrm{MHz}$, Chloroform- $d$ ) $\delta: 7.37 \sim 7.31(\mathrm{~m}, 2 \mathrm{H}), 7.19(\mathrm{~s}, 1 \mathrm{H}), 6.90 \sim 6.85$ (m, 2H), 3.83 (s, 3H); ${ }^{13} \mathrm{C}$ NMR (101 MHz, Chloroform- $d$ ) $\delta: 159.8,135.2,130.2,113.6,96.5,55.3$; MS (EI) $\mathrm{m} / \mathrm{z}: 386$ ([M] $\left.]^{+}\right)$; IR (film) $v: 3064,2927,2833,1604,1500,1294$, $1251,1174,1153,1109,1029,852,829,769,624,594 \mathrm{~cm}^{-1}$.

(E)-1-(1,2-二碘乙烯基)-3-氟苯 $(\mathbf{2 h})^{[18]}$ : 黄色油状液 体, $44 \mathrm{mg}$, 产率 59\%. ${ }^{1} \mathrm{H}$ NMR (400 MHz, Chloroform- $d$ ) $\delta: 7.34$ (td, $J=7.9,5.8 \mathrm{~Hz}, 1 \mathrm{H}), 7.31$ (s, 1H), 7.14 (d, $J=$ $7.8 \mathrm{~Hz}, 1 \mathrm{H}), 7.09 \sim 6.98(\mathrm{~m}, 2 \mathrm{H}) ;{ }^{13} \mathrm{C}$ NMR $(101 \mathrm{MHz}$, Chloroform- $d$ ) $\delta: 162.2$ (d, $J=248.5 \mathrm{~Hz}), 145.0$ (d, $J=8.1$ $\mathrm{Hz}), 130.1$ (d, $J=8.4 \mathrm{~Hz}), 124.2(\mathrm{~d}, J=3.0 \mathrm{~Hz}), 116.1$ (d, $J=21.1 \mathrm{~Hz}), 115.6(\mathrm{~d}, J=22.9 \mathrm{~Hz}), 93.9,81.8 ;{ }^{19} \mathrm{~F}$ NMR (282 MHz, Chloroform- $d$ ) $\delta$ : $-111.9(\mathrm{td}, J=8.7,5.6 \mathrm{~Hz})$; MS (EI) $m / z: 294$ ([M] $]^{+}$); IR (film) v: 3064, 2920, 2850, 1581, 1477, 1427, 1261, 1203, 1122, 941, 875, 767, 694, $611 \mathrm{~cm}^{-1}$.

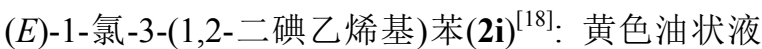


体, $47 \mathrm{mg}$, 产率 60\%. ${ }^{1} \mathrm{H}$ NMR $(400 \mathrm{MHz}$, Chloroform-d) $\delta: 7.36 \sim 7.27(\mathrm{~m}, 4 \mathrm{H}), 7.25 \sim 7.21(\mathrm{~m}, 1 \mathrm{H}) ;{ }^{13} \mathrm{C}$ NMR (101 MHz, Chloroform-d) $\delta$ : 144.7, 134.1, 129.7, 129.1, 128.5, 126.7, 93.8, 82.1; MS (EI) $m / z: 389$ ([M] $]^{+}$); IR (film) $v$ : 3292, 3064, 1562, 1471, 1408, 1238, 1195, 1159, 1093, 1078, 881, 844, 785, 736, 719, 686, $607 \mathrm{~cm}^{-1}$.

(E)-1-(1,2-二碘乙烯基)-4-氟苯 $(\mathbf{2} \mathbf{j})^{[18]}$ : 黄色油状液 体, $61 \mathrm{mg}$, 产率 $81 \% .{ }^{1} \mathrm{H}$ NMR (400 MHz, Chloroform- $d$ ) $\delta: 7.39 \sim 7.32(\mathrm{~m}, 2 \mathrm{H}), 7.27(\mathrm{~s}, 1 \mathrm{H}), 7.10 \sim 7.02(\mathrm{~m}, 2 \mathrm{H})$; ${ }^{13} \mathrm{C}$ NMR (101 MHz, Chloroform- $d$ ) $\delta: 162.5$ (d, $J=250.8$ $\mathrm{Hz}), 139.1$ (d, $J=3.5 \mathrm{~Hz}), 130.6$ (d, $J=8.5 \mathrm{~Hz}), 115.5$ (d, $J=22.0 \mathrm{~Hz}), 94.8,81.5 ;{ }^{19} \mathrm{~F}$ NMR (282 MHz, Chloroform- $d$ ) $\delta:-111.0$ (tt, $J=8.3,4.8 \mathrm{~Hz}$ ); MS (EI) $m / z: 374$ $\left([\mathrm{M}]^{+}\right)$; IR (film) $v: 3062,1598,1591,1498,1402,1230$, 1157, 1093, 1014, 858, 835, 783, 624, $594 \mathrm{~cm}^{-1}$.

(E)-1-溴-4-(1,2-二碘乙烯基)苯 $(2 \mathbf{k})^{[21 b]}$ : 白色固体, $72 \mathrm{mg}$, 产率 82\%. m.p. 55.6 57.4 ${ }^{\circ} \mathrm{C}$ (lit. ${ }^{[2 \mathrm{~b}]} 63 \sim$ $\left.64{ }^{\circ} \mathrm{C}\right) ;{ }^{1} \mathrm{H}$ NMR (400 MHz, Chloroform- $d$ ) $\delta: 7.53 \sim 7.48$ (m, 2H), 7.29 (s, 1H), 7.26 7.22 (m, 2H); ${ }^{13} \mathrm{C}$ NMR (101 MHz, Chloroform- $d$ ) $\delta: 142.0,131.7,130.2,123.1,94.5$, 81.7; MS (EI) $m / z: 434$ ([M] $]^{+}$); IR (film) v: 3061, 1897, 1573, 1475, 1390, 1244, 1151, 1103, 1068, 1008, 943, 844, $815,779,719,597,553 \mathrm{~cm}^{-1}$.

(E)-4-(1,2-二碘乙烯基)-1,1'-联苯(2I) ${ }^{[22]}$ : 白色固体, $62 \mathrm{mg}$, 产率 70\%. m.p. $96.3 \sim 98.0{ }^{\circ} \mathrm{C}\left(\right.$ lit. $\left.{ }^{[22} 96 \sim 98{ }^{\circ} \mathrm{C}\right)$; ${ }^{1} \mathrm{H}$ NMR (400 MHz, Chloroform- $d$ ) $\delta$ : 7.62 (dd, $J=11.2$, $8.0 \mathrm{~Hz}, 4 \mathrm{H}), 7.50 \sim 7.45(\mathrm{~m}, 4 \mathrm{H}), 7.40(\mathrm{~d}, J=7.1 \mathrm{~Hz}, 1 \mathrm{H})$, $7.31(\mathrm{~s}, 1 \mathrm{H}) ;{ }^{13} \mathrm{C}$ NMR (101 MHz, Chloroform- $d$ ) $\delta: 141.8$, 141.7, 140.2, 129.1, 128.9, 127.7, 127.1, 127.1, 96.1, 80.7; MS (EI) $\mathrm{m} / z$ : 432 ([M] $]^{+}$); IR (film) $v$ : 3064, 1672, 1604, 1483, 1400, 1263, 1151, 1004, 852, 829, 769, 738, 690, $597 \mathrm{~cm}^{-1}$.

(E)-3-（(1,2-二碘乙烯基)吡啶(2m) ${ }^{[18]}$ : 淡黄色固体, $27 \mathrm{mg}$, 产率 $40 \%$. m.p. $67.0 \sim 68.7{ }^{\circ} \mathrm{C} ;{ }^{1} \mathrm{H}$ NMR $(400$ $\mathrm{MHz}$, Chloroform- $d$ ) $\delta: 8.69 \sim 8.61(\mathrm{~m}, 1 \mathrm{H}), 8.56(\mathrm{~d}, J=$ $3.7 \mathrm{~Hz}, 1 \mathrm{H}), 7.67$ (dt, $J=7.9,1.9 \mathrm{~Hz}, 1 \mathrm{H}), 7.42(\mathrm{~s}, 1 \mathrm{H})$, 7.32 (dd, $J=7.9,4.9 \mathrm{~Hz}, 1 \mathrm{H}) ;{ }^{13} \mathrm{C}$ NMR $(101 \mathrm{MHz}$, Chloroform- $d$ ) $\delta$ : 149.6, 149.2, 139.3, 136.3, 123.2, 91.5, 83.2; MS (EI) $m / z$ : 357; IR (film) $v: 3059,2922,1573,1467$, 1408, 1247, 1192, 1157, 1120, 1024, 846, 806, 707, 601.

(E)- $N$-(3-(1,2-二碘乙烯基)苯基)-2,2,3,3-四甲基环丙 烷-1-羧酰胺 (2n): 白色固体, $60 \mathrm{mg}$, 产率 $61 \%$. m.p. 151.3 152.1 ${ }^{\circ} \mathrm{C}$; ${ }^{1} \mathrm{H}$ NMR (400 MHz, Chloroform- $d$ ) $\delta$ : $7.53(\mathrm{~d}, J=6.9 \mathrm{~Hz}, 1 \mathrm{H}), 7.34(\mathrm{~s}, 1 \mathrm{H}), 7.28(\mathrm{~s}, 1 \mathrm{H}), 7.23(\mathrm{~d}$, $J=7.3 \mathrm{~Hz}, 1 \mathrm{H}), 7.20$ (s, 1H), 6.99 (d, $J=7.6 \mathrm{~Hz}, 1 \mathrm{H}), 1.29$ (s, 6H), 1.17 (s, 6H); ${ }^{13} \mathrm{C}$ NMR (101 MHz, Chloroform- $d$ ) $\delta: 170.1,143.7,138.5,129.1,123.5,120.0,119.0,95.5$, 38.5, 29.7, 23.8, 16.7; IR (film) v: 3298, 2922, 2864, 1658, 1587, 1541, 1479, 1423, 1377, 1300, 1224, 1197, 1112, 798, $698 \mathrm{~cm}^{-1}$; HRMS (ESI) calcd for $\mathrm{C}_{16} \mathrm{H}_{19} \mathrm{I}_{2} \mathrm{NONa}$ $[\mathrm{M}+\mathrm{Na}]^{+}$517.9454, found 517.9445.

(E)-1,2-二碘-2-甲基苯乙烯 $(20)^{[18]}$ : 黄色油状液体, $46 \mathrm{mg}$, 产率 $61 \% .{ }^{1} \mathrm{H}$ NMR (400 MHz, Chloroform- $d$ ) $\delta$ : $7.41 \sim 7.23(\mathrm{~m}, 5 \mathrm{H}), 2.83(\mathrm{~s}, 3 \mathrm{H}) ;{ }^{13} \mathrm{C}$ NMR $(101 \mathrm{MHz}$, Chloroform- $d$ ) $\delta: 148.1,128.4,128.4,128.2,96.3,95.5$, 40.2; MS (EI) $m / z: 370$ ([M] $]^{+}$); IR (film) $v: 3055,2910$, 1487, 1440, 1371, 1072, 1029, 1002, 987, 844, 754, 692, $632,576 \mathrm{~cm}^{-1}$.

(E)-1,2-二碘-2-苯乙基苯乙烯 $(\mathbf{2} \mathbf{p})^{[23]}$ : 黄色油状液 体, $78 \mathrm{mg}$, 产率 $85 \% .{ }^{1} \mathrm{H}$ NMR (400 MHz, Chloroform- $d$ ) $\delta: 7.30(\mathrm{~d}, J=5.6 \mathrm{~Hz}, 6 \mathrm{H}), 7.23(\mathrm{dd}, J=14.9,7.4 \mathrm{~Hz}, 2 \mathrm{H})$, $7.13(\mathrm{~d}, J=7.9 \mathrm{~Hz}, 2 \mathrm{H}), 3.17 \sim 3.08(\mathrm{~m}, 2 \mathrm{H}), 2.97 \sim 2.90$ $(\mathrm{m}, 2 \mathrm{H}) ;{ }^{13} \mathrm{C}$ NMR (101 MHz, Chloroform- $d$ ) $\delta: 148.1$, $139.8,129.0,128.4,128.4,128.3,128.2,126.4,103.5$, 95.7, 52.5, 34.3; MS (EI) $m / z: 460$ ([M] ${ }^{+}$); IR (film) $v$ : 3028, 1598, 1489, 1454, 1442, 1340, 1076, 968, 914, 754, $690,580 \mathrm{~cm}^{-1}$.

(E)-1,2-二碘-2-苯乙基-(4-甲基)苯乙烯(2q)：黄色油 状液体, $60 \mathrm{mg}$, 产率 64\%. ${ }^{1} \mathrm{H}$ NMR $(400 \mathrm{MHz}$, Chloroform- $d$ ) $\delta: 7.28 \sim 7.25(\mathrm{~m}, 3 \mathrm{H}), 7.19(\mathrm{~s}, 2 \mathrm{H}), 7.09$ (d, $J=$ $7.9 \mathrm{~Hz}, 2 \mathrm{H}), 7.00$ (d, $J=8.1 \mathrm{~Hz}, 2 \mathrm{H}), 3.07$ (dd, $J=9.4,6.3$ $\mathrm{Hz}, 2 \mathrm{H}), 2.90$ (dd, $J=9.4,6.3 \mathrm{~Hz}, 2 \mathrm{H}), 2.29$ (s, $3 \mathrm{H}) ;{ }^{13} \mathrm{C}$ NMR (101 MHz, Chloroform- $d$ ) $\delta$ : 145.3, 139.8, 138.2, 129.1, 129.0, 128.4, 128.2, 126.4, 103.2, 96.1, 52.7, 34.3, 21.4; IR (film) v: 3024, 2920, 1504, 1496, 1452, 1180, $1022,812,758,746,698,586 \mathrm{~cm}^{-1}$; HRMS (EI) calcd for $\mathrm{C}_{17} \mathrm{H}_{16} \mathrm{I}_{2} 473.9342$, found 473.9334 .

(E)-1,2-二碘-4-苯基-1-丁烯 $(2 \mathbf{r})^{[18]}$ : 棕色油状液体, $57 \mathrm{mg}$, 产率 74\%. ${ }^{1} \mathrm{H}$ NMR (300 MHz, Chloroform-d) $\delta$ : $7.45 \sim 7.19(\mathrm{~m}, 5 \mathrm{H}), 6.91(\mathrm{~s}, 1 \mathrm{H}), 2.89(\mathrm{~s}, 4 \mathrm{H}) ;{ }^{13} \mathrm{C} \mathrm{NMR}$ (101 MHz, Chloroform-d) $\delta:$ 139.6, 128.8, 128.4, 126.4, 102.4, 80.1, 46.8, 34.1; MS (EI) $m / z: 384$ ([M] ${ }^{+}$); IR (film) $v: 3062,3024,2943,1602,1494,1452,1215,1163,1004$, $902,773,746,696,590,559 \mathrm{~cm}^{-1}$.

(E)-1,2-二碘-3-甲基-4-(叔丁基)苯基-1-丁烯(2s): 无 色透明液体, $57 \mathrm{mg}$, 产率 $40 \% .{ }^{1} \mathrm{H}$ NMR $(400 \mathrm{MHz}$, Chloroform-d) $\delta: 7.36 \sim 7.28(\mathrm{~m}, 2 \mathrm{H}), 7.18(\mathrm{~d}, J=8.3 \mathrm{~Hz}$, $2 \mathrm{H}), 6.82(\mathrm{~s}, 1 \mathrm{H}), 2.74 \sim 2.47(\mathrm{~m}, 3 \mathrm{H}), 1.32(\mathrm{~s}, 9 \mathrm{H}), 1.01$ (d, $J=6.1 \mathrm{~Hz}, 3 \mathrm{H}) ;{ }^{13} \mathrm{C}$ NMR (101 MHz, Chloroform-d) $\delta$ : 149.2, 135.5, 129.0, 125.1, 114.8, 45.9, 42.1, 34.4, 31.4, 
20.2; IR (film) v: 2962, 2866, 1903, 1514, 1454, 1361, 1269, 1220, 1109, 1049, 929, 856, 827, 771, 623, 565 $\mathrm{cm}^{-1}$; HRMS (EI) calcd for $\mathrm{C}_{15} \mathrm{H}_{20} \mathrm{I}_{2}$ 453.9654, found 453.9648

(E)-3,4-二碘-3-丁烯-1-(3,4-二甲氧基苯甲酸酯)(2t): 白色固体, $75 \mathrm{mg}$, 产率 $77 \%$. m.p. $67.7 \sim 69.4{ }^{\circ} \mathrm{C} ;{ }^{1} \mathrm{H}$ NMR (400 MHz, Chloroform- $d$ ) $\delta: 7.71$ (dd, $J=8.5,2.0$ $\mathrm{Hz}, 1 \mathrm{H}), 7.55$ (d, $J=2.0 \mathrm{~Hz}, 1 \mathrm{H}), 7.02$ (s, 1H), 6.88 (d, $J=$ $8.5 \mathrm{~Hz}, 1 \mathrm{H}), 4.46(\mathrm{t}, J=6.1 \mathrm{~Hz}, 2 \mathrm{H}), 3.92(\mathrm{~s}, 6 \mathrm{H}), 3.02(\mathrm{t}$, $J=6.1 \mathrm{~Hz}, 2 \mathrm{H}) ;{ }^{13} \mathrm{C}$ NMR (101 MHz, Chloroform- $d$ ) $\delta$ : $166.1,153.1,148.5,123.9,122.3,112.1,110.2$, 98.1, 82.1, 62.0, 56.1, 56.0, 44.2; IR (film) v: 2956, 2835, 1712, 1598, $1514,1463,1417,1346,1292,1269,1220,1176,1134$, 1109, 1024, 763, $632 \mathrm{~cm}^{-1}$; HRMS (ESI) calcd for $\mathrm{C}_{13} \mathrm{H}_{15} \mathrm{I}_{2} \mathrm{O}_{4}[\mathrm{M}+\mathrm{H}]^{+}$488.9054, found 488.9056 .

$(E)-1,2$-二碘癸烯 $(\mathbf{2 u})^{[18]}$ : 无色透明液体, $57 \mathrm{mg}$, 产 率 73\%. ${ }^{1} \mathrm{H}$ NMR (400 MHz, Chloroform- $d$ ) $\delta: 6.80$ (s, $1 \mathrm{H}), 2.55 \sim 2.44(\mathrm{~m}, 2 \mathrm{H}), 1.54(\mathrm{t}, J=7.4 \mathrm{~Hz}, 2 \mathrm{H}), 1.40 \sim$ $1.24(\mathrm{~m}, 11 \mathrm{H}), 0.89$ (s, 3H); ${ }^{13} \mathrm{C}$ NMR (101 MHz, Chloroform-d) $\delta: 104.5,78.9,44.6,31.8,29.4,29.2,28.2,28.1$, 22.7, 14.1; MS (EI) $m / z: 392\left([\mathrm{M}]^{+}\right)$; IR (film) v: 2922, 2852, 1463, 1377, 1217, 1114, 767, 721, $569 \mathrm{~cm}^{-1}$.

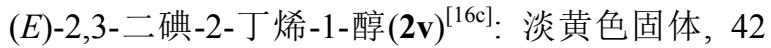
$\mathrm{mg}$, 产率 65\%. m.p. 93.5 94.6 ${ }^{\circ} \mathrm{C} ;{ }^{1} \mathrm{H}$ NMR (400 MHz, Chloroform- $d$ ) $\delta: 4.42(\mathrm{~d}, J=1.0 \mathrm{~Hz}, 2 \mathrm{H}), 2.65$ (d, $J=1.0$ $\mathrm{Hz}, 3 \mathrm{H}), 2.08(\mathrm{~s}, 1 \mathrm{H}) ;{ }^{13} \mathrm{C}$ NMR (101 MHz, Chloroform- $d$ ) $\delta: 102.6,95.1,76.7,40.1$; MS (EI) $m / z: 324\left([\mathrm{M}]^{+}\right)$; IR (film) $v: 3207,2920,1712,1448,1371,1226,1068,1010$, $908,752,626 \mathrm{~cm}^{-1}$.

(E)-5,6-二碘-5-己烯酸 $(2 \mathrm{w})$ : 白色固体, $44 \mathrm{mg}$, 产率 $60 \%$. m.p. 69.7 71.5 ${ }^{\circ} \mathrm{C} .{ }^{1} \mathrm{H}$ NMR (400 MHz, Chloroform- $d$ ) $\delta: 6.90(\mathrm{~s}, 1 \mathrm{H}), 2.61(\mathrm{t}, J=7.3 \mathrm{~Hz}, 2 \mathrm{H}), 2.42(\mathrm{t}$, $J=7.5 \mathrm{~Hz}, 2 \mathrm{H}), 1.91$ (q, $J=7.4 \mathrm{~Hz}, 2 \mathrm{H}) ;{ }^{13} \mathrm{C}$ NMR $(101$ $\mathrm{MHz}$, Chloroform- $d$ ) $\delta$ : 179.2, 102.3, 80.5, 43.7, 32.1, 23.1; IR (film) v: 2947, 1697, 1456, 1311, 1234, 1159, 1012, 948, 856, 812, 767, $567 \mathrm{~cm}^{-1}$; HRMS (EI) calcd for $\mathrm{C}_{6} \mathrm{H}_{8} \mathrm{I}_{2} \mathrm{O}_{2} 365.8614$, found 365.8611 .

(E)-2-氯- $N$-(3-(1,2-二碘乙烯基) 苯基)烟酰胺 $(\mathbf{2} \mathbf{x})$ : 黄色固体, $43 \mathrm{mg}$, 产率 50\%. m.p. 163.3 164.8 ${ }^{\circ} \mathrm{C} ;{ }^{1} \mathrm{H}$ NMR (400 MHz, DMSO-d $) \delta: 9.94(\mathrm{~s}, 1 \mathrm{H}), 7.70$ (d, $J=$ $4.6 \mathrm{~Hz}, 1 \mathrm{H}), 7.27$ (d, $J=7.5 \mathrm{~Hz}, 1 \mathrm{H}), 6.87$ (s, 1H), 6.80 (d, $J=11.8 \mathrm{~Hz}, 2 \mathrm{H}), 6.77 \sim 6.70(\mathrm{~m}, 1 \mathrm{H}), 6.56(\mathrm{t}, J=7.9 \mathrm{~Hz}$, $1 \mathrm{H}), 6.22(\mathrm{~d}, J=7.6 \mathrm{~Hz}, 1 \mathrm{H}) ;{ }^{13} \mathrm{C} \mathrm{NMR}(101 \mathrm{MHz}$, DMSO- $\left.d_{6}\right) \delta: 163.6,150.5,146.4,144.1,138.7,138.2$, $132.9,129.1,123.6,123.1,119.6,118.8,96.6,84.8$; IR (film) v: 3361, 3199, 2920, 2850, 1658, 1581, 1546, 1471, 1398, 1319, 1259, 1130, 1066, 881, 802, 754, $698 \mathrm{~cm}^{-1}$; HRMS (ESI) calcd for $\mathrm{C}_{14} \mathrm{H}_{9} \mathrm{ClI}_{2} \mathrm{~N}_{2} \mathrm{ONa}[\mathrm{M}+\mathrm{Na}]^{+}$ 532.8390 , found 532.8379 .

(E)- $N$-(3-(1,2-二碘乙烯基)苯基)-4-甲基苯磺酰胺 (2y): 白色固体，76 mg，产率 $72 \%$. m.p. $119.3 \sim$ $121.0{ }^{\circ} \mathrm{C} ;{ }^{1} \mathrm{H}$ NMR (400 MHz, Chloroform-d) $\delta: 7.87$ (d, $J=8.3 \mathrm{~Hz}, 2 \mathrm{H}), 7.59$ (s, 1H), $7.43 \sim 7.31(\mathrm{~m}, 4 \mathrm{H}), 7.27 \sim$ $7.16(\mathrm{~m}, 3 \mathrm{H}), 2.50(\mathrm{~s}, 3 \mathrm{H}) ;{ }^{13} \mathrm{C}$ NMR $(101 \mathrm{MHz}$, Chloroform-d) $\delta: 144.2,144.1,136.7,135.7,129.8,129.5,127.4$, 125.2, 121.9, 121.1, 94.6, 81.6, 81.6, 21.6, 21.6; IR (film) $v: 3250,2922,1585,1465,1396,1328,1159,1091,968$, 877, 812, 700, $671 \mathrm{~cm}^{-1}$; HRMS (ESI) calcd for $\mathrm{C}_{15} \mathrm{H}_{13} \mathrm{I}_{2-}$ $\mathrm{NO}_{2} \mathrm{SNa}[\mathrm{M}+\mathrm{Na}]^{+}$547.8654, found 547.8644.

甲基 $((S)-2-(($ 叔丁氧羰基)氨基)-3-(4- $((E)-1,2-$ 二碘 乙烯基)苯基)丙酰基)- $L$-缴氨酸盐(2z)：淡黄色油状液 体, $67 \mathrm{mg}$, 产率 37\%. ${ }^{1} \mathrm{H}$ NMR (400 MHz, Chloroform- $d$ ) $\delta: 7.23 \sim 7.09(\mathrm{~m}, 5 \mathrm{H}), 6.38(\mathrm{~d}, J=7.6 \mathrm{~Hz}, 1 \mathrm{H}), 4.94(\mathrm{~s}$, $1 \mathrm{H}), 4.37(\mathrm{dd}, J=8.1,5.3 \mathrm{~Hz}, 1 \mathrm{H}), 4.34 \sim 4.15(\mathrm{~m}, 1 \mathrm{H})$, 3.62 (s, 3H), 2.98 (qd, $J=14.0,6.9 \mathrm{~Hz}, 2 \mathrm{H}), 2.03$ (dq, $J=$ 13.0, $6.6 \mathrm{~Hz}, 1 \mathrm{H}), 1.32$ (s, 9H), 0.77 (dd, $J=13.9,6.8 \mathrm{~Hz}$, $6 \mathrm{H}) ;{ }^{13} \mathrm{C}$ NMR (101 MHz, Chloroform-d) $\delta: 171.8,170.9$, $155.4,141.5,137.7,129.4,128.9,95.9,57.2,52.2,37.7$, 31.1, 29.7, 28.3, 18.9, 17.8; IR (film) v: 3315, 2964, 2926, 1743, 1681, 1654, 1531, 1365, 1251, 1170, 1022, 761, 597 $\mathrm{cm}^{-1}$; HRMS (ESI) calcd for $\mathrm{C}_{22} \mathrm{H}_{30} \mathrm{I}_{2} \mathrm{~N}_{2} \mathrm{O}_{5} \mathrm{Na}[\mathrm{M}+\mathrm{Na}]^{+}$ 697.0142, found 697.0141.

4-((E)-1,2-二碘乙烯基)苯甲酸 $((1,2),(3,4)-$ 二丙酮叉6-半乳糖)酯(2aa): 无色浆液， $60 \mathrm{mg}$ ，产率 $46 \% .{ }^{1} \mathrm{H}$ NMR (400 MHz, Chloroform- $d$ ) $\delta: 8.05$ (d, $J=8.3 \mathrm{~Hz}$, 2H), $7.41(\mathrm{~d}, J=8.3 \mathrm{~Hz}, 2 \mathrm{H}), 7.33$ (s, 1H), 5.57 (d, $J=4.9$ $\mathrm{Hz}, 1 \mathrm{H}), 4.66$ (dd, $J=7.9,2.3 \mathrm{~Hz}, 1 \mathrm{H}), 4.52$ (dd, $J=11.5$, $4.9 \mathrm{~Hz}, 1 \mathrm{H}), 4.44(\mathrm{dd}, J=11.5,7.5 \mathrm{~Hz}, 1 \mathrm{H}), 4.38 \sim 4.29$ $(\mathrm{m}, 2 \mathrm{H}), 4.18(\mathrm{t}, J=5.5 \mathrm{~Hz}, 1 \mathrm{H}), 1.52(\mathrm{~s}, 3 \mathrm{H}), 1.48(\mathrm{~s}, 3 \mathrm{H})$, $1.36(\mathrm{~s}, 3 \mathrm{H}), 1.34$ (s, 3H); ${ }^{13} \mathrm{C}$ NMR (101 MHz, Chloroform-d) $\delta: 165.7,147.5,130.2,129.9,128.5,109.7,108.8$, $96.3,94.3,81.9,71.1,70.7,70.5,66.0,64.1,26.0,26.0$, 24.9, 24.5; IR (film) v: 2985, 2931, 1720, 1382, 1273, 1211, 1166, 1103, 1068, 1006, 896, 711, $599 \mathrm{~cm}^{-1}$; HRMS (ESI) calcd for $\mathrm{C}_{21} \mathrm{H}_{24} \mathrm{I}_{2} \mathrm{O}_{7} \mathrm{Na}[\mathrm{M}+\mathrm{Na}]^{+} 664.9509$, found 664.9508 .

(1S,2S,4S)-1,7,7-三甲基双环[2.2.1]庚-2-取代-3-((E)1,2-二碘-3-甲氧基-1-丙烯基)苯甲酸酯(2ab): 无色液体， $100 \mathrm{mg}$, 产率 89\%. ${ }^{1} \mathrm{H}$ NMR (400 MHz, Chloroform- $d$ ) $\delta$ : 7.99 (dt, $J=7.5,1.7 \mathrm{~Hz}, 1 \mathrm{H}), 7.91$ (d, $J=1.9 \mathrm{~Hz}, 1 \mathrm{H})$, 
$7.49 \sim 7.38(\mathrm{~m}, 2 \mathrm{H}), 5.11(\mathrm{ddd}, J=9.9,3.5,2.1 \mathrm{~Hz}, 1 \mathrm{H})$, $4.43(\mathrm{~s}, 2 \mathrm{H}), 3.46(\mathrm{~s}, 3 \mathrm{H}), 2.57 \sim 2.38(\mathrm{~m}, 1 \mathrm{H}), 2.12(\mathrm{ddd}$, $J=13.3,9.4,4.4 \mathrm{~Hz}, 1 \mathrm{H}), 1.90 \sim 1.68(\mathrm{~m}, 2 \mathrm{H}), 1.55 \sim 1.20$ $(\mathrm{m}, 2 \mathrm{H}), 1.13(\mathrm{dd}, J=13.8,3.5 \mathrm{~Hz}, 1 \mathrm{H}), 1.05 \sim 0.86(\mathrm{~m}$, $9 \mathrm{H}) ;{ }^{13} \mathrm{C}$ NMR (101 MHz, Chloroform-d) $\delta: 165.9,147.5$, $132.5,131.2,129.5,128.6,102.8,96.0,83.9,80.9,57.8$, $49.1,47.9,45.0,36.9,28.1,27.4,19.7,18.9,13.7$; IR (film) $v: 2954,2883,1716,1579,1303,1286,1199,1109$, 1018, 756, $700 \mathrm{~cm}^{-1}$; HRMS (EI) calcd for $\mathrm{C}_{21} \mathrm{H}_{26} \mathrm{I}_{2} \mathrm{O}_{3}$ 579.9971 , found 579.9975 .

4-碘-3-(4-戊基苯基)-1 $H$-异戊烯-1-酮( $\left.\mathbf{S}^{\prime}\right)$ : 白色固 体, $65 \mathrm{mg}$, 产率 78\%. m.p. 78.1 79.4 ${ }^{\circ} \mathrm{C} ;{ }^{1} \mathrm{H}$ NMR (400 $\mathrm{MHz}$, Chloroform- $d$ ) $\delta: 8.29$ (dd, $J=7.9,1.4 \mathrm{~Hz}, 1 \mathrm{H}), 7.88$ $(\mathrm{d}, J=8.0 \mathrm{~Hz}, 1 \mathrm{H}), 7.85 \sim 7.76(\mathrm{~m}, 1 \mathrm{H}), 7.62(\mathrm{~d}, J=8.0$ $\mathrm{Hz}, 2 \mathrm{H}), 7.59 \sim 7.51(\mathrm{~m}, 1 \mathrm{H}), 7.28(\mathrm{~d}, J=7.9 \mathrm{~Hz}, 2 \mathrm{H})$, $2.80 \sim 2.60(\mathrm{~m}, 2 \mathrm{H}), 1.77 \sim 1.57(\mathrm{~m}, 2 \mathrm{H}), 1.41 \sim 1.30(\mathrm{~m}$, $4 \mathrm{H}), 0.99 \sim 0.83(\mathrm{~m}, 3 \mathrm{H}) ;{ }^{13} \mathrm{C}$ NMR (101 MHz, Chloroform-d) $\delta$ : 161.6, 155.0, 145.4, 138.3, 135.6, 132.5, 131.5, $129.9,129.7,129.0,128.0,120.2,35.8,31.5,30.8,22.5$, 14.0; IR (film) v: 2920, 1905, 1732, 1602, 1506, 1469, 1226, 1076, 1024, 943, 848, 763, 688, $640 \mathrm{~cm}^{-1}$; HRMS (ESI) calcd for $\mathrm{C}_{20} \mathrm{H}_{20} \mathrm{IO}_{2}[\mathrm{M}+\mathrm{H}]^{+}$419.0502, found 419.0500 .

\section{辅助材料(Supporting Information) 产物的 ${ }^{1} \mathrm{H} \mathrm{NMR}$ 、 ${ }^{13} \mathrm{C}$ NMR、 ${ }^{19} \mathrm{~F}$ NMR 核磁共振谱图. 这些材料可以免费 从本刊网站(http://sioc-journal.cn/)上下载.}

\section{References}

[1] (a) Seechurn, C. C. J.; Kitching, M. O.; Colacot, T. J.; Snieckus, V. Angew. Chem., Int. Ed. 2012, 51, 5062.

(b) Piontek, A.; Bisz, E.; Szostak, M. Angew. Chem., Int. Ed. 2018, $57,11116$.

(c) King, A. O.; Yasuda, N. In Organometallics in Process Chemistry, Springer, Berlin, 2004, pp. 205 245.

(d) Ian, W. Ph.D. Dissertation, Durham University, Durham, 2011.

(e) Zeng, X.; Liu, S.; Yang, Y.; Yang, Yi; Hammond, G. B.; Xu, B. Chem 2020, 6, 1018.

[2] (a) Zani, L.; Dessì, A.; Franchi, D.; Calamante, M.; Reginato, G.; Mordini, A. Coord. Chem. Rev. 2019, 392, 177.

(b) Kawabata, K.; Saito, M.; Osaka, I.; Takimiya, K. J. Am. Chem. Soc. 2016, 138, 7725.

(c) Furukawa, S.; Yasuda, T. J. Mater. Chem. A 2019, 7, 14806.

(d) Chen, L.; Zeng, M.; Weng, C.; Tan, S.; Shen, P. ACS Appl. Mater. Interfaces 2019, 11, 48134.

(e) Tovar, J. D. Synthesis 2011, 2387.

(f) Olla, T.; Ibraikulov, O. A.; Ferry, S.; Boyron, O.; Méry, S.; Heinrich, B.; Heiser, T.; Lévêque, P.; Leclerc, N. Macromolecules 2019, 52, 8006 .

[3] (a) Atzrodt, J.; Derdau, V.; Fey, T.; Zimmermann, J. Angew. Chem., Int. Ed. 2007, 46, 7744.

(b) Liu, C.; Chen, Z.; Su, C.; Zhao, X.; Gao, Q.; Ning, G.-H.; Zhu,
H.; Tang, W.; Leng, K.; Fu, W.; Tian, B.; Peng, X.; Li, J.; Xu, Q.-H.; Zhou, W.; Loh, K. P. Nat. Commun. 2018, 9, 80.

[4] (a) Welch, M. J.; Redvanly, C. S. In The Hand Book of Radiopharmaceuticals: Radiochemistry and Applications, Ed.: Finn, R., Wiley, New York, 2003, p. 423.

(b) Hallouard, F.; Anton, N.; Choquet, P.; Constantinesco, A.; Vandamme, T. Biomaterials 2010, 31, 6249.

(c) Zimprich, F.; Rath, J.; Mauritz, M.; Zulehner, G.; Hilfer, Eva.; Cetin, H.; Kasprian, G.; Auff, E. J. Neurol. 2017, 264, 1209.

[5] (a) Gkotsi, D. S.; Ludewig, H.; Sharma, S. V.; Connolly, J. A.; Dhaliwal, J.; Wang, Y.; Unsworth, W. P.; Taylor, R. J. K.; McLachlan, M. M. W.; Shanahan, S.; Naismith, J. H.; Goss, R. J. M. Nat. Chem. 2019, 11, 1091.

(b) Butler, A.; Sandy, M. Nature 2009, 460, 848.

(c) Latham, J.; Brandenburger, E.; Shepherd, A.; Menon, B. R. K.; Micklefield, J. Chem. Rev. 2018, 118, 232.

[6] Li, Y.; Mou, T.; Lu, L.; Jiang, X. Chem. Commun., 2019, 55, 14299.

[7] (a) Banerjee, S.; Khatri, H.; Balasanthiran, V.; Koodali, R. T.; Sereda, G. Tetrahedron 2011, 67, 5717.

(b) Ranu, B. C.; Adak, L.; Chattopadhyay, K. J. Org. Chem. 2008, 73, 5609 .

(c) Kimura, T.; Nishimura, Y.; Ishida, N.; Momochi, H.; Yamashita, H.; Satoh, T. Tetrahedron Lett. 2013, 54, 1049.

[8] (a) Bao, Y.; Yang, X.; Zhou, Q.; Yang, F. Org. Lett. 2018, 20, 1966. (b) Lin, Y.; Lu, G.; Cai, C.; Yi, W. Org. Lett. 2015, 17, 3310.

[9] Li, J. H.; Tang, S. Synth. Commun. 2005, 35, 105.

[10] (a) Liang, Y.; Tao, L.-M.; Zhang, Y.-H.; Li, J.-H. Synthesis 2008, 3988.

(b) Raff, G.; Belot, S.; Balme, G.; Monteiro, N. Org. Biomol. Chem. 2011, 9, 1474.

[11] Schuh, K.; Glorius, F. Synthesis 2007, 2297.

[12] Shen, G.; Yang, B.; Huang, X.; Hou, Y.; Gao, H.; Cui, J.; Cui, C.; Zhang, T. J. Org. Chem. 2017, 82, 3798.

[13] Woodward, S.; Ackermann, M.; Ahirwar, S. K.; Burroughs, L.; Garrett, M. R.; Ritchie, J.; Shine, J.; Tyril, B.; Simpson, K.; Woodward, P. Chem. Eur. J. 2017, 3, 7819.

[14] (a) Sun, A.; Lauher, J. W.; Goroff, N. S. Science 2006, 312, 1030. (b) DeCicco, R. C.; Luo, L.; Goroff, N. S. Acc. Chem. Res. 2019, $52,2080$.

[15] Terent'ev, A. O.; Borisov, D. A.; Krylov, I. B.; Nikishin, G. I. Synth. Commun. 2007, 37, 3151.

[16] (a) Dolbier, W. R.; Duan, J.-X.; Chen, Q.-Y. J. Org. Chem. 1998, 63, 9486.

(b) Li, J.-H.; Tang, S. Synth. Commun. 2005, 35, 105.

(c) Su, L.; Lei, C.-Y.; Fan, W.-Y.; Liu, L.-X. Synth. Commun. 2011, 41,1200 .

[17] (a) Li, J.-H.; Xie, Y.-X.; Yin, D.-L. Green Chem. 2002, 4, 505. (b) Larson, S.; Luidhardt, T.; Kabalka, G. W.; Pagni, R. M. Tetrahedron Lett. 1988, 29, 35.

(c) Song, S.; Li, X.; Wei, J.; Wang, W.; Zhang, Y.; Ai, L.; Zhu, Y.; Shi, X.; Zhang, X.; Jiao, N. Nat. Catal. 2020, 3, 107.

[18] Banothu, R.; Peraka, S.; Kodumuri, S.; Chevella, D.; Gajula, K. S.; Amrutham, V.; Yennamaneni, D. R.; Nama, N. New J. Chem. 2018, $42,17879$.

[19] Stavber, S.; Stavber, G.; Iskra, J.; Zupan, M. Adv. Synth. Catal. 2008, 350, 2921.

[20] Guo, C.-C.; Jiang, Q.; Wang, J.-Y. Synthesis 2015, 47, 2081.

[21] (a) Liu, Y.; Maruoka, K.; Huang, D.-Y.; Huang, J. J. Org. Chem. 2017, 82,11865

(b) Kashyap, S.; Rao D. S.; Reddy, T. R. Org. Biomol. Chem. 2018, 16, 1508.

[22] Madabhushi, S.; Jillella, R.; Mallu, K. K. R.; Golada, K. R.; Vangipuram, V. S. Tetrahedron Lett. 2013, 54, 3993.

[23] Yang, F.; Jin, T.; Bao, M.; Yamamoto, Y. Chem. Commun. 2011, 47, 4013. 